\title{
25 Research Soure \\ Realization of a polarization-insensitive optical frequency-domain reflectometer using an I/Q homodyne detection
}

\author{
Alin Jderu \\ S.C. NanoPRO START MC \\ Marcelo A. Soto \\ Universidad Técnica Federico Santa María, \\ Marius Enachescu \\ University POLITEHNICA Bucharest \\ Dominik Ziegler ( $\nabla$ dominik.foslab@gmail.com ) \\ S.C. NanoPRO START MC
}

\section{Research}

Keywords: Optical frequency-domain reflectometry, distributed optical fiber sensing, temperature sensing

Posted Date: January 26th, 2021

DOI: https://doi.org/10.21203/rs.3.rs-150679/v1

License: @ (i) This work is licensed under a Creative Commons Attribution 4.0 International License.

Read Full License 


\section{Abstract}

We report on the development and implementation of an optical frequency-domain reflectometer (OFDR) sensing platform. OFDR allows to measure changes in strain and temperature using optical fibers with a length of several tens of meters with very high spatial resolution. We discuss the operation principles and challenges to implement an OFDR system using optical homodyne detection based on a dualpolarization $90^{\circ}$ optical hybrid. Our setup exhibits polarization and phase diversity, fully automated data acquisition and data processing using a LabVIEW-based implemented software environment. Using an optical hybrid enables to discriminate phase, amplitude and polarization by interfering the Rayleigh scatter signal and a local oscillator with four $90^{\circ}$ phase stepped interferences between the two signals. Without averaging and a fast acquisition time of $230 \mathrm{~ms}$, our preliminary results show a spatial resolution of $5 \mathrm{~cm}$ and a temperature resolution of about 0.1 Kelvin on a $3 \mathrm{~m}$-long fiber.

\section{Introduction}

Over the last decades the field of optical fiber sensing has grown immensely. The application fields of the technology range from shape sensing, pipeline and electrical transmission line monitoring, structural health monitoring, or intrusion detection for perimeter security applications [1]. Unlike optical time-domain reflectometry (OTDR), optical frequency-domain reflectometry (OFDR) interrogates a sensing optical fiber in the frequency domain using a tunable continuous-wave (CW) laser, which enables high spatial resolutions while securing high signal-to-noise ratios. OFDR in optical fibers has first been introduced by W. Eickhoff and R. Ulrich in 1981 [2], and over time many variants have evolved [3, 4]. In a typical OFDR system a tunable laser source is swept over a broad wavelength band close to $1550 \mathrm{~nm}$. While one part of the light is sent into a sensing fiber, another part is used as an optical local oscillator to enable coherent detection. The strain and temperature information is contained in the optical phase of the Rayleigh scattering, and their measurement is carried out by tracking the changes of the Rayleigh scattering pattern. The spatial distribution of the reflected light along the fiber is obtained using both the Fourier and inverse Fourier transforms of the measured coherent Rayleigh backscattered signal.

In this paper, we give a detailed description of the development of an engineered OFDR sensing platform using a robust in-phase/quadrature (I/Q) homodyne detection with phase and polarization diversity. The paper also describes relevant aspects related to the signal processing, including a robust methodology to measure large temperature (or strain) changes with high precision. The current manuscript can serve as a guideline to build reliable and robust high-resolution OFDR sensors.

\section{Materials And Methods}

\section{Principle of Rayleigh-based OFDR sensing}

Rayleigh-based OFDR sensing makes use of the stable, random interference pattern caused by coherent Rayleigh scattering generated along an optical fiber. Refractive index changes caused by external 
perturbations, like temperature or strain changes, induce a phase shift of the Rayleigh scattered light at the local position of the perturbation. The Rayleigh backscattered signal is measured by an optical coherent detector, in which the light backscattered from the sensing fiber is made to interfere with an optical local oscillator. OFDR sensors can measure the Rayleigh phase shifts induced by those external perturbations utilizing a sweeping laser source. Compared to time-domain approaches, in which short pulses of light propagate along the fiber, OFDR uses a continuous-wave laser with an optical frequency that is linearly swept over time. Since the light is continuous-wave, a high signal-to-noise ratio (SNR) can be obtained while securing sharp spatial resolution in the measurand profile that is retrieved from the changes in the Rayleigh interference patterns as the wavelength is swept. While the OFDR experimental setup is relatively simple, high-quality components are required for the implementation. To obtain high spatial resolution, the optical frequency of the laser light must be swept in a wide spectral range. The optical frequency sweeping can be obtained for instance using external modulation, in which the optical carrier of a continuous wave laser is intensity modulated by a constant-amplitude radiofrequency (RF) signal whose frequency is linearly swept over a certain frequency range either stepwise (step-frequency method) [5] or continuously (sweep frequency method) [6]. After launching this frequency-swept light into the sensing fiber, the Rayleigh backscattered signal generated in the fiber is detected as a function of modulation frequency. This corresponds to the frequency response of the optical fiber Rayleigh scattering, and hence the Fourier transform of the measured signals must be computed to obtain the local information in the distance domain. Considering a sufficiently fast sampling rate, the resolution of the OFDR sensor is given by , where is the speed of light, is the refractive index of the optical fiber and is the frequency sweep range [2].

\section{Homodyne Detection in OFDR}

In the most basic implementation of an OFDR system, as shown in Figure 1a, the Rayleigh backscattered light from the sensing fiber (S) is combined with the local oscillator (LO) with an optical coupler. Then the orthogonal polarization states of the optical signals are split using a polarizing beam splitter (PBS), whose outputs are connected to two independent photodetectors. To provide polarization diversity a polarization controller must be used, as shown in Figure 1a. This implementation is affected if the polarization of the local oscillator drifts over time. It also requires a proper frequency offset between the local oscillator and the Rayleigh backscattered light to provide fast variations of the phase at an intermediate frequency to allow for reliable optical phase estimation. A more robust alternative is to use a I/Q homodyne detector, making use of a dual-polarization $90^{\circ}$ optical hybrid, as shown in Figure 1b [7]. This dual-polarization hybrid takes the Rayleigh scattering signal and optical local oscillator, and provides four outputs separated by $90^{\circ}$ for each orthogonal polarization.

Optical $90^{\circ}$ hybrids are passive optical devices that can, as shown in Figure 2a be realized in free space optics or as monolithically as arrayed waveguide gratings where fiber implementations are possible (Figure 2b). In such configuration, planar arrayed waveguide gratings can be realized in silicon [8], lithium niobate [9], or silicon on insulator materials [10]. However, in all these configurations control of the phase is required [11]. Complementary outputs from the hybrid ( $0^{\circ}$ and $180^{\circ}$ ports, and $90^{\circ}$ and $270^{\circ}$ ports) are 
fed to the inputs of a pair of balanced detectors, resulting electrical output signals in quadrature which are obtained for each polarization [12]. This way, the $90^{\circ}$ optical hybrid can provide both polarization and phase diversity for reliable optical phase detection [7]. In optical communication systems this approach is used for decades already to provide highly accurate phase measurements [13].

The phase difference between complementary pairs of output ports in the $90^{\circ}$ hybrid $\left(A_{1} \& A_{2}, A_{3} \& A_{4}\right.$ $A_{5} \& A_{6}$, or $\left.A_{7} \& A_{8}\right)$ is always $180^{\circ}$. Each of these pairs of optical signals are fed into a balance photodetector, which are used to eliminate the baseband components of the coherent detection process. One balanced photodetector detects the Rayleigh electrical field component reaching the photodetector front-end in-phase (I) with the local oscillator, while the other photodetector obtains the Rayleigh electric field component in quadrature (Q) with the local oscillator. Since the state of polarization of the Rayleigh backscattered light could easily change along the sensing optical fiber and reach the input of the $90^{\circ}$ hybrid with random state, polarization diversity is provided by replicating the I and Q component detection for the orthogonal state of polarization, making use of the other two balanced photodetectors. This way, the detection of the Rayleigh backscattered signal becomes insensitive to fluctuation of its state of polarization.

\section{Optoelectronic Implementation}

In our OFDR implementation, we used a tunable laser (8mW, Phoenix 1400, Luna) which is set to sweep a spectral range of $23 \mathrm{~nm}$ (from $1541 \mathrm{~nm}$ to $1564 \mathrm{~nm}$ ) using a tuning rate of $100 \mathrm{~nm} / \mathrm{s}$. This leads to a measurement time of 0.23 s per acquired trace, corresponding to a fiber sampling rate of about $4.3 \mathrm{~Hz}$. This external cavity laser is driven by a circuit designed for low noise and highly linear and mode hop free wavelength sweeping required for stable fiber sensing applications. A homodyne detection using a dualpolarization $90^{\circ}$ optical hybrid (Kylia, $\mathrm{COH} 28$ ), and four balanced detectors have been used to obtain polarization and phase diversity. The used balanced photodetectors (Thorlabs, PDB470C) have a bandwidth from $D C$ to $400 \mathrm{MHz}$, a common mode rejection ratio of $>25 \mathrm{~dB}$, and a noise equivalent power of $8 \mathrm{pW} \cdot \mathrm{Hz}^{-0.5}$. For data acquisition, the output signals from the photodetectors are connected to four parallel channels of a 16-bit analog-to-digital converter card (National Instruments, PCle-6376) with a sampling rate of $3.5 \mathrm{MS} / \mathrm{s}$ per channel. Lastly, the sensing fiber used in this experiment is a standard single-mode optical fiber of $3 \mathrm{~m}$ long. The light reflection at the far end of the fiber is eliminated by coiling up the fiber end with a radius of curvature smaller than $1 \mathrm{~cm}$. Similar results have been obtained placing with a commercial attenuator at the end of the fiber.

\section{Results}

\section{Extracting the Rayleigh Frequency Shift}


The different data processing steps for OFDR detection are as shown in Figure 3. The voltage signal generated by each of the four balanced photodetectors shown in Figure $1 \mathrm{~b}$ corresponds to the difference of the photo-detected signals ( $I_{\mathrm{Ai}}$, with $i=1$ to 8$)$ for each pair of orthogonal outputs of the $90^{\circ}$ hybrid. These signals can be respectively represented as $A=I_{A 1}-I_{A 2}, B=$ $I_{A 3}-I_{A 4}, C=I_{A 5}-I_{A 6}$ and $D=I_{A 7}-I_{A 8}$. Based on the four digitized signals, we first calculate the amplitude of the Rayleigh backscattering electric field by using the inphase $(I)$ and quadrature $(Q)$ components as follows $I=A+C$ and $Q=B+D$. This way the amplitude of the Rayleigh scattering field is calculated as $\sqrt{I^{2}+Q^{2}}$.

Since measurements are obtained by sweeping the laser wavelength, this Rayleigh scattering amplitude corresponds to the amplitude of the frequency response of the fiber. Using FFT, the Rayleigh response along the sensing fiber can be obtained. In order to obtain temperature and strain variations, the local Rayleigh spectrum is then obtained by calculating the inverse FFT (IFFT) of windowed Rayleigh response over the fiber location. Then the obtained local Rayleigh spectrum is compared to a reference spectrum measured at known temperature and strain conditions. This comparison is performed at each fiber location by calculating a circular normalized cross-correlation of the actual local Rayleigh spectrum and its respective reference [14]. This way, the resulting correlation peak frequency will give information of the local fiber refractive index change, which is proportional to the local temperature and strain variation. It is worth noting that this cross-correlation must be circular (sometimes also called periodic), i.e. data points at the end of the sampled spectrum array must be fed into the beginning of the spectrum while calculating the spectral cross-correlation. Note that if a conventional correlation is used, a triangular shaped pattern would be obtained, from which it will be difficult to accurately identify the correlation peak frequency, containing the useful local information. The circular cross-correlation, however, does not have such behavior, but results in a flat background with a very noticeable correlation peak that leads to more reliable peak detection results.

Synchronisation of the data acquisition, triggering of the laser wavelength sweeping is implemented using LabVIEW. The user interface consists of four parts: an interface for the data acquisition, the signal processing to extract the measurand information, a data visualization interface, and a data recording section.

\section{Experimental Setup}

To evaluate proper functioning of our OFDR device we immerse about $1 \mathrm{~m}$ of a standard optical fiber into a water bath, which is continuously heated up in a stepwise manner. This simple setup is shown in Figure $4 a$ and $4 b$, which allows us to obtain a uniform temperature distribution along the immersed fiber. With a 
hotplate and a thermometer we control and monitor the temperature of the liquid over time as we gradually raise the water temperature. For a precise comparison we monitor the water temperature with a resolution of $10 \mathrm{mK}$, which is good enough to discriminate the different steps.

\section{Temperature Measurements}

While the water temperature is increased during the experiment data measured by our OFDR setup is recorded using our LabVIEW interface. Using the measurement at $25^{\circ} \mathrm{C}$ as a reference, the resulting correlation maps for 12 different temperatures ranging from $25^{\circ} \mathrm{C}$ to $37^{\circ} \mathrm{C}$ are shown in Figure $5 \mathrm{a}$. As expected, a clear correlation peak results where the fiber was not heated, for instance around a distance of $150 \mathrm{~cm}$, the correlation of the measured Rayleigh spectrum with itself at the $25^{\circ} \mathrm{C}$ condition. Where the fiber is immersed in water (approximately from $25 \mathrm{~cm}$ to $100 \mathrm{~cm}$ ) the temperature rises, and the Rayleigth frequency shift of the immersed optical fiber section also increases. The cross-correlation peak amplitude decreases with the increased temperature. In Figure $5 b$, we show the retrieved temperature shift obtained by the OFDR system for all conditions depicted in Figure 5a. Results show that for small temperature changes up to a few degrees Celsius with respect to the reference, we can see a clear detection of the peak location resulting in a reliable measured temperature profile (see for instance $30^{\circ} \mathrm{C}$ and $35^{\circ} \mathrm{C}$ ); however, generally for larger temperature shifts the peak detection and retrieved temperature profile becomes unreliable.

In order to measure large temperature changes (or equivalently large strain variations), the correlation peak can be obtained updating the reference trace when the correlation peak shifts more than a given threshold value. Updating the reference every time the correlation frequency shift varies more than 1.2 $\mathrm{GHz}$ (corresponding to $1 \mathrm{~K}$ temperature shift approximately), the differential temperature maps for the 12 previously shown temperatures are recalculated and shown in Figure $6 a$, while Figure $6 \mathrm{~b}$ illustrates the resulting differential temperature profiles. Results demonstrate a more reliable temperature profile extraction, compared to the case reported in Figure 5.

Knowing the reference temperature profile and tracking the Rayleigh frequency shift for each of the reference updates, the distributed profile of the absolute temperature can be obtained as reported in Figure 7a. The temperature resolution is obtained by the standard deviation of the temperature profile at fiber location not affected by temperature changes, resulting in a resolution of $0.1 \mathrm{~K}$; while the spatial resolution is $5 \mathrm{~cm}$, being in this case limited by the spatial sampling rate.

In Figure $7 \mathrm{~b}$ we show the average of the frequency shifts found between a distance of $40 \mathrm{~cm}$ and $80 \mathrm{~cm}$, where the fiber was fully immersed in water. The frequency shifts are displayed against the actually measured temperatures using a precise thermometer. The result shows good linearity, from linear fitting we observe a sensitivity of $1.2 \mathrm{GHz} / \mathrm{K}$, which is expected and typical for silica based optical fibers.

\section{Conclusion}


We developed a short-range distributed optical fiber sensing platform based on OFDR method for temperature and strain monitoring. We have designed and realized an integrated OFDR sensing platform, that contains all opto-electronic components in a compact enclosure. The use of a 90 degrees optical hybrid provides the system with polarization and phase diversity, making the sensor much more reliable, as fine twists or motion of the fiber that change the light polarization no longer affect strain or temperature measurements.

The implemented OFDR system has led to preliminary results that demonstrate the proper working capability of the system, verifying a temperature resolution of $0.1 \mathrm{~K}$ and a spatial resolution of $5 \mathrm{~cm}$, at the designed temporal sampling rate of $4.3 \mathrm{~Hz}$ (fiber sampling interval of $0.23 \mathrm{~s}$ ). Note that the spatial resolution is in this case limited by the spatial sampling rate of the system which has been set to 5 $\mathrm{cm} /$ point, given by the sliding window length of the IFFT used to calculate the local Rayleigh scattering spectrum. The number of points used in this IFFT is 2000 points which could be reduced to improve the spatial sampling interval, with the cost of degrading the temperature resolution accordingly. Thus, for instance, if the spatial sampling interval is improved down to $1 \mathrm{~cm}$, then the temperature resolution would result in $0.5 \mathrm{~K}$, for the temporal sampling rate of $4.3 \mathrm{~Hz}$. Another alternative is to compromise the temporal sampling rate, so by averaging consecutive traces a much higher spatial and/or temperature resolution could be obtained at slower interrogation rates.

\section{Declarations}

\section{Availability of data and materials}

The datasets used and/or analysed during the current study are available from the corresponding author on reasonable request.

\section{Competing interests}

The authors declare no conflict of interest.

\section{Funding}

This research was funded by Romanian Ministry of Education and Research, \#34/01.09.2016, ID: P_37_788, MySMIS: 103364; European Regional Development Fund, Competitiveness Operational Program; ANID Chilean National Agency for Research and Development, Basal Project FB0008 and Fondequip Regular 1200299.

\section{Authors' contributions}

Conceptualization, M.A.S., A.J. and D.Z.; methodology, M.A.S., M.E. and D.Z.; software, M.A.S. and A.J.; validation, M.A.S., and D.Z.; formal analysis, M.A.S. and D.Z.; investigation, M.A.S., A.J., and D.Z.; resources, M.E.; data curation, M.A.S. and A.J.; writing- original draft preparation, M.A.S. and D.Z.; writing 
-review and editing, M.A.S., M.E. and D.Z.; visualization, M.A.S. and D.Z.; supervision, M.A.S. and M.E.; project administration, M.E. and D.Z.; funding acquisition, M.E. and D.Z.

\section{Acknowledgements}

Not Applicable.

\section{References}

[1] Lu, P, Lalam, N, Badar, M, Liu, B, Chorpening, BT, Buric, MP, Ohodnicki, PR: Distributed optical fiber sensing: Review and perspective. Appl. Phys. Rev. 6, 041302 (2019). https://doi.org/10.1063/1.5113955

[2] Eickhoff, W, Ulrich, R: Optical frequency domain reflectometry in single-mode fiber. Appl. Phys. Lett. 39, 693 (1981). https://doi.org/10.1063/1.92872

[3] Froggatt, M, Moore, J: High-spatial-resolution distributed strain measurement in optical fiber with Rayleigh scatter. Appl. Opt. 37, 1735-1740 (1998). tps://doi.org/10.1364/A0.37.001735

[4] Ding, Z, Wang, C, Liu, K, Jiang, J, Yang, D, Pan, G, Pu, Z, Liu, T: Distributed Optical Fiber Sensors Based on Optical Frequency Domain Reflectometry: A review. Sensors. 18, 1072 (2018). https://doi.org/10.3390/s18041072

[5] Nakayama, J, lizuka, K, Nielsen, J: Optical fiber fault locator by the step frequency method. Appl. Opt. 26, 440-443 (1987). https://doi.org/10.1364/A0.26.000440

[6] Dolfi, DW, Nazarathy, M, Newton, SA: 5-mm-resolution optical-frequency-domain reflectometry using a coded phase-reversal modulator. Opt. Lett. 13, 678-680 (1988). tps://doi.org/10.1364/OFS.1988.WDD7

[7] Arbel, D, Eyal, A: Dynamic optical frequency domain reflectometry. Opt. Expr. 22(8), 8823 (2014). https://doi.org/10.1364/OE.22.008823.

[8] Sakamaki, Y, Yamazaki, H, Mizuno, T, Goh, T, Nasu, Y, Hashimoto, T, Kamei, S, Hattori, K, Takahashi, H: One-chip integrated dual polarization optical hybrid using silica-based planar lightwave circuit technology. European Conference and Exhibition on Optical Communication (IEEE), pp. 1-2, 2009.

[9] Hoffman, D, Heidrich, H, Wenke, G, Langenhorst, R, Dietrich, E: Integrated optics eight-port $90^{\circ}$ hybrid on LiNb03. J. Light. Technol. 7(5), 794-798 (1989).https://doi.org/10.1109/50.19116

[10] Faralli, S, Meloni, G, Gambini, F, Klamkin, J, Potì, L, Contestabile, G: A compact silicon coherent receiver without waveguide crossing. IEEE Photonics J. 7(4), 1-6 (2015).

https://doi.org/10.1109/JPHOT.2015.2456632

[11] Seimetz, M, Weinert, CM: Options, feasibility and availability of $2 \times 490^{\circ}$ hybrids for coherent optical systems. J. Light. Technol. 24(3), 1317-1322 (2006). https://doi.org/10.1109/JLT.2005.863251 
[12] Hartog, AH: An Introduction to Distributed Optical Fibre Sensors. CRC Press. Boca Raton, FL, USA (2017).

[13] Hodgkinson, TG, Harmon, RA, Smith, DW: Demodulation of optical DPSK using in-phase and quadrature detection. Electron. Lett. 21(19), 867 (1985). https://doi.org/10.1049/el:19850612

[14] Koyamada, Y, Imahama, M, Kubota, K, Hogari, K: Fiber-Optic Distributed Strain and Temperature Sensing With Very High Measurand Resolution Over Long Range Using Coherent OTDR. J. Light. Technol. 27(9), 1142-1146, May1, (2009). doi: 10.1109/JLT.2008.928957.

\section{Figures}
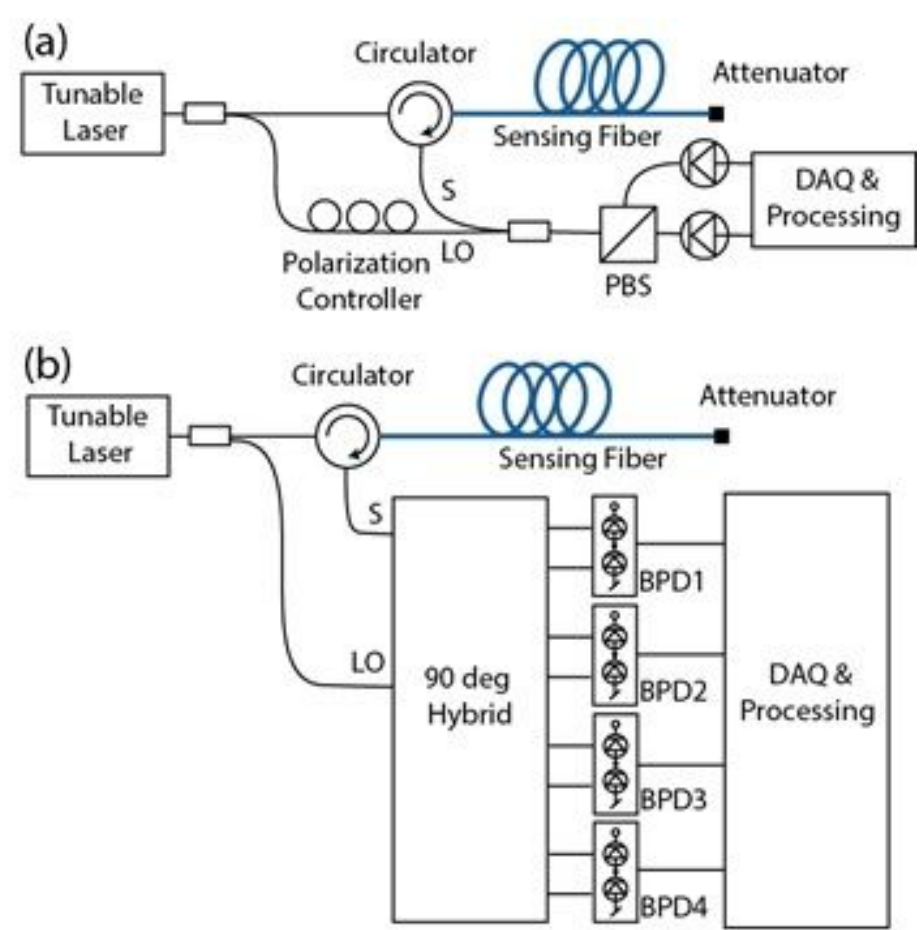

\section{Figure 1}

(a) Optoelectronic schematic of a typical OFDR setup using a polarizing beam splitter (PBS) for polarization diversity. (b) Schematic of our OFDR implementation using a full I/Q optical homodyne detector, based on a dual-polarization $90^{\circ}$ optical hybrid, four balanced photodetectors (BPD) and a data acquisition (DAQ) with four acquisition channels. 

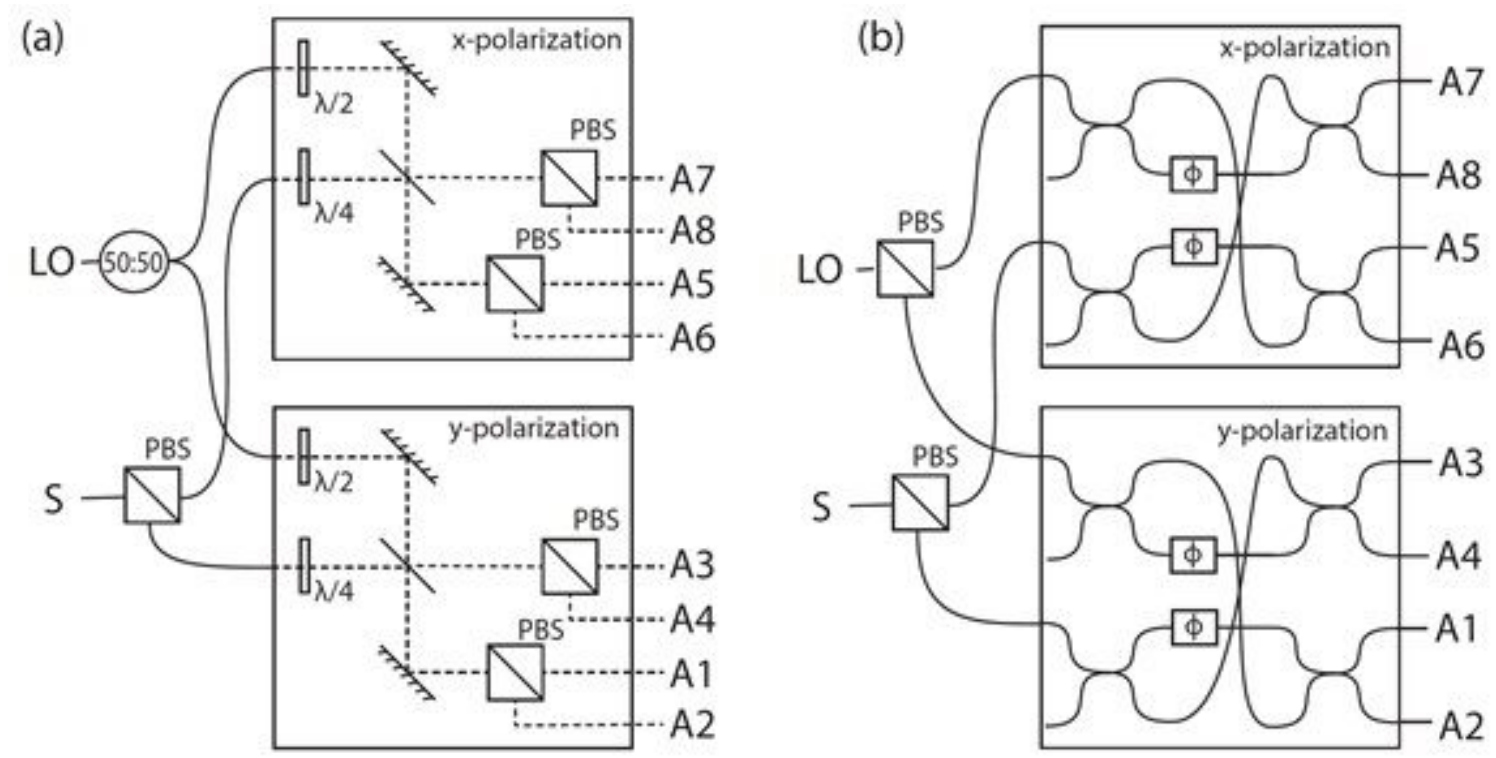

Figure 2

Schematic representation of different 90 degree optical hybrid implementations. (a) free space implementation consisting of half-wave plates $(\lambda / 2)$, quarter-wave plates $(\lambda / 4)$, polarizing beam splitters (PBS), beam combiners and mirrors. (b) fiber optical implementation using phase shifters (PS) and combiners.

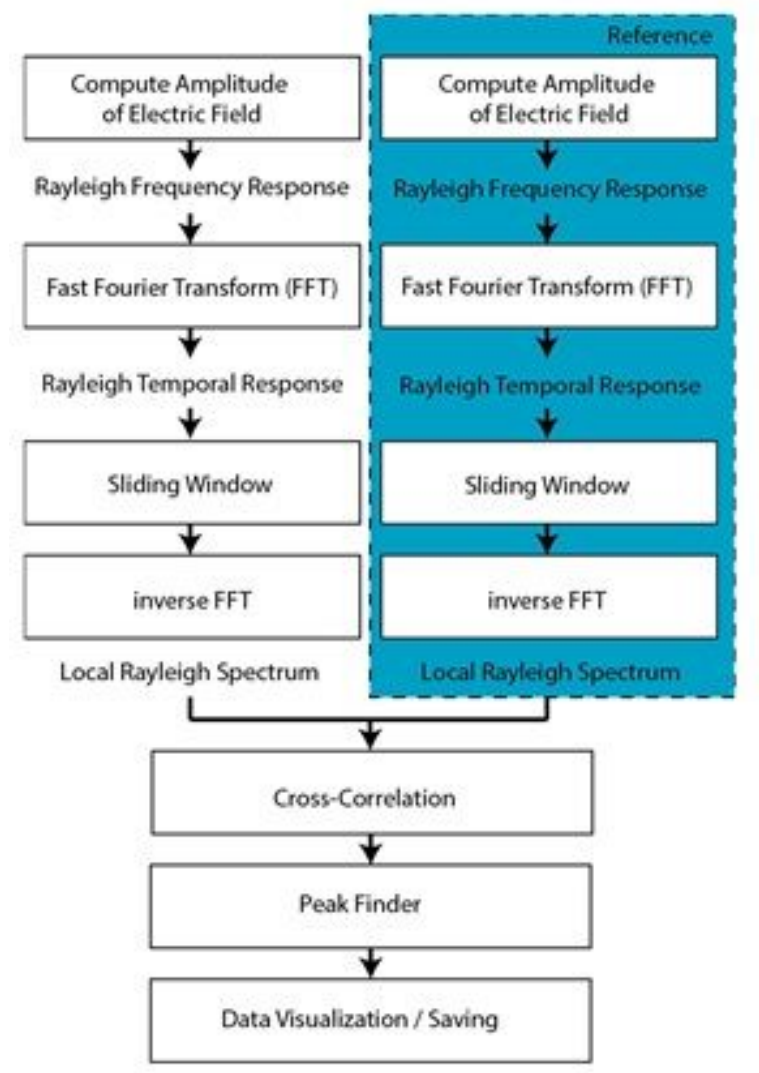

Figure 3 
Flow diagram of the data processing to extract the Rayleigh frequency shift from the output of balanced photodetector signals.

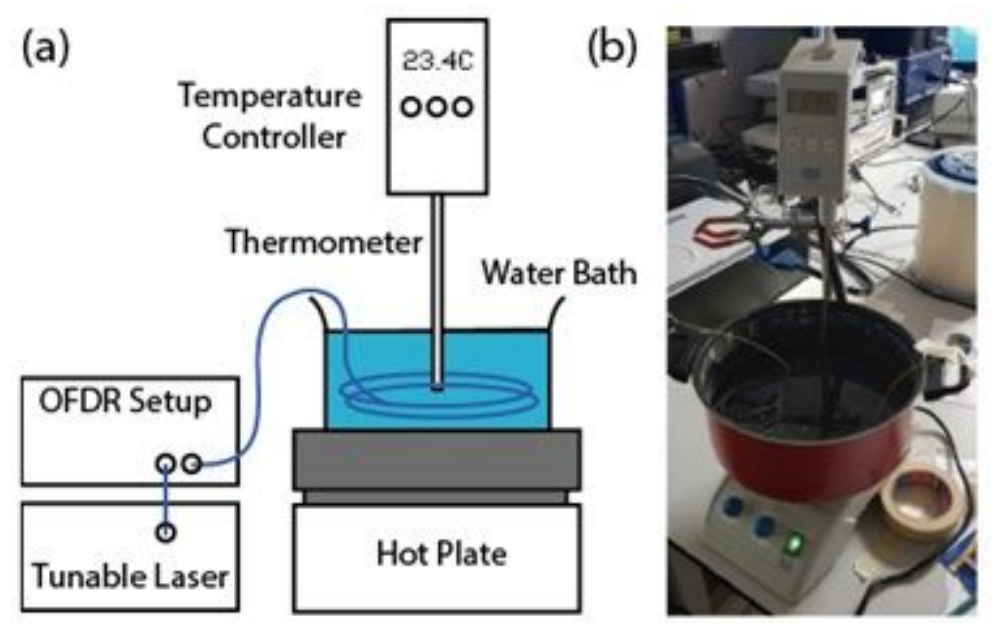

\section{Figure 4}

Experimental setup for temperature sensing (a) schematic and (b) photograph of the simple experimental setup for temperature sensing.

(a)
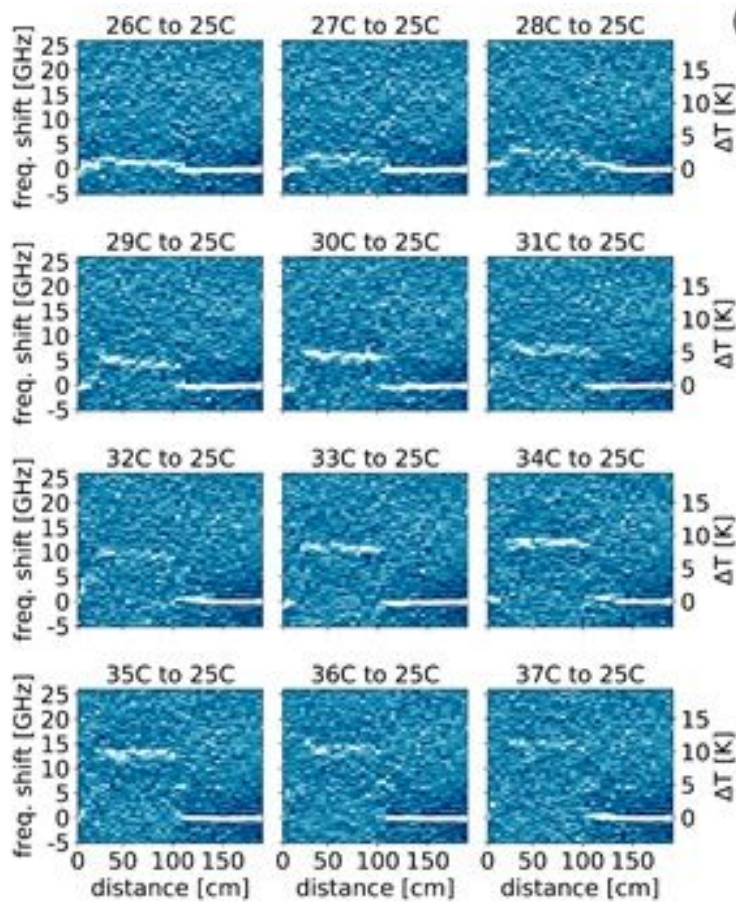

(b)
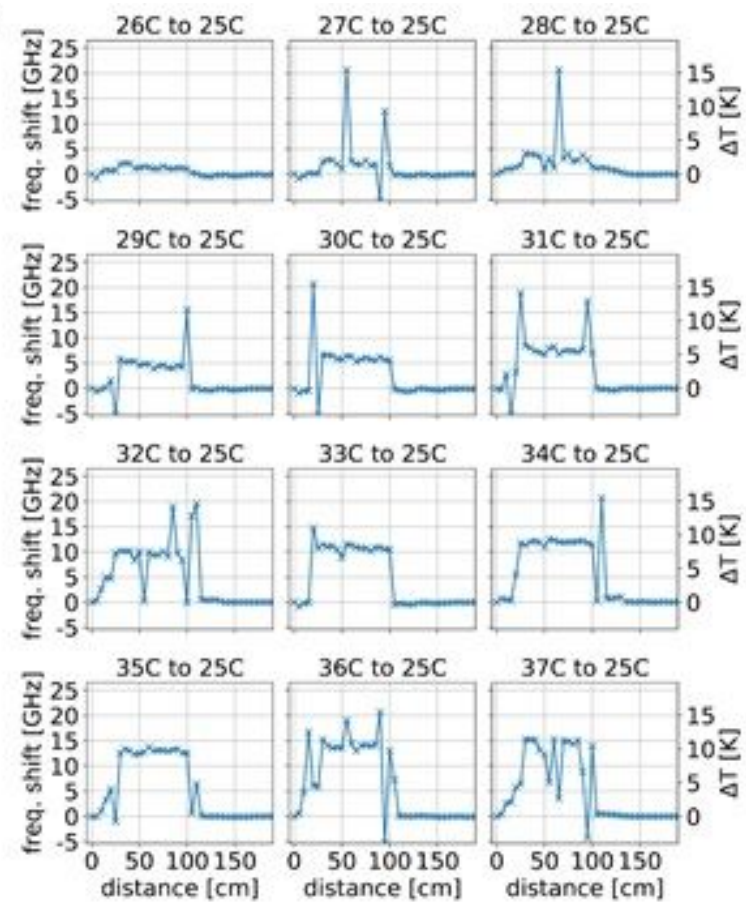

\section{Figure 5}

(a) Retrieved distributed differential correlation maps for 12 temperatures ranging from $25^{\circ} \mathrm{C}$ to $37^{\circ} \mathrm{C}$. (b) Retrieved temperature profiles show that the correlation peak detection becomes unreliable with larger temperature differences as the correlation amplitude fades. 
(a)
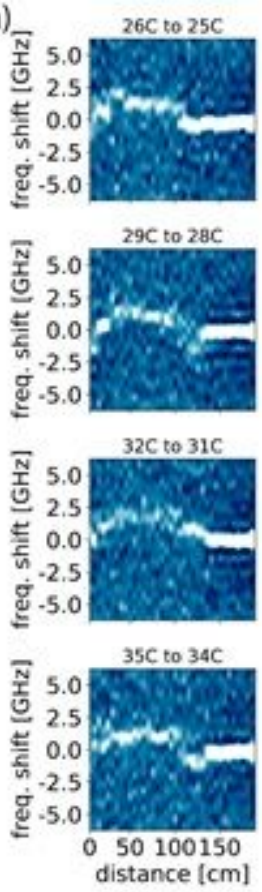

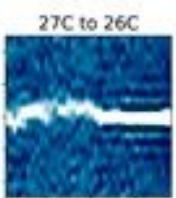

$30 \mathrm{C}$ to $29 \mathrm{C}$
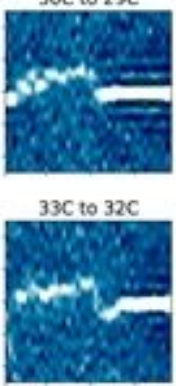

$36 \mathrm{C}$ to $35 \mathrm{C}$

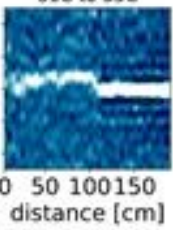

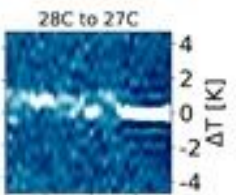

$31 \mathrm{C}$ to $30 \mathrm{C}$

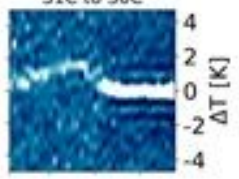

$34 \mathrm{C}$ to $33 \mathrm{C}$

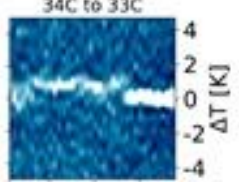

$37 \mathrm{C}$ to $36 \mathrm{C}$

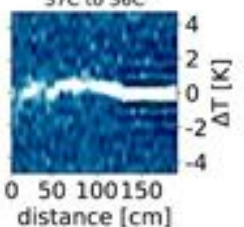

(b)
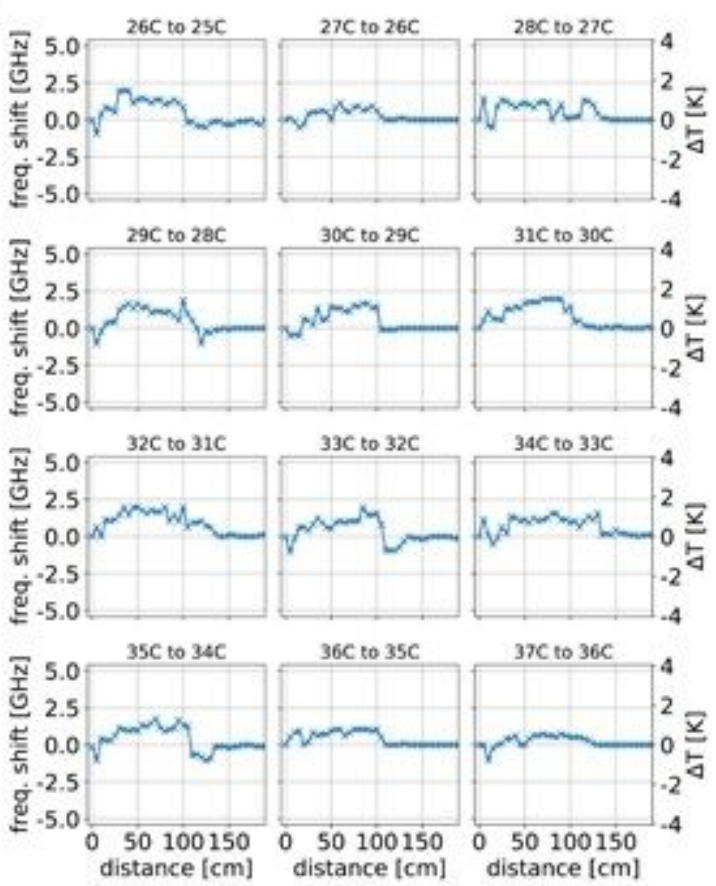

Figure 6

(a) Retrieved distributed differential temperature maps for 16 temperatures ranging from $25^{\circ} \mathrm{C}$ to $36^{\circ} \mathrm{C}$, when correlating each data set with the previous one. (b) Retrieved differential temperature profiles.

(a)

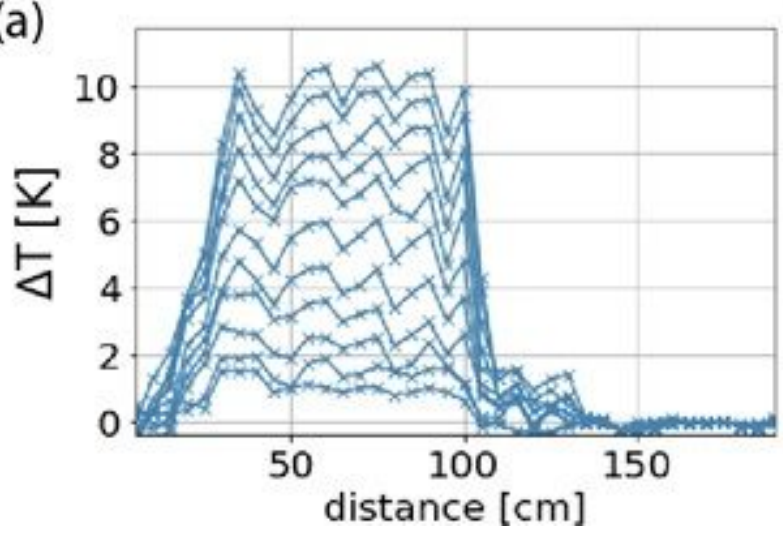

(b)

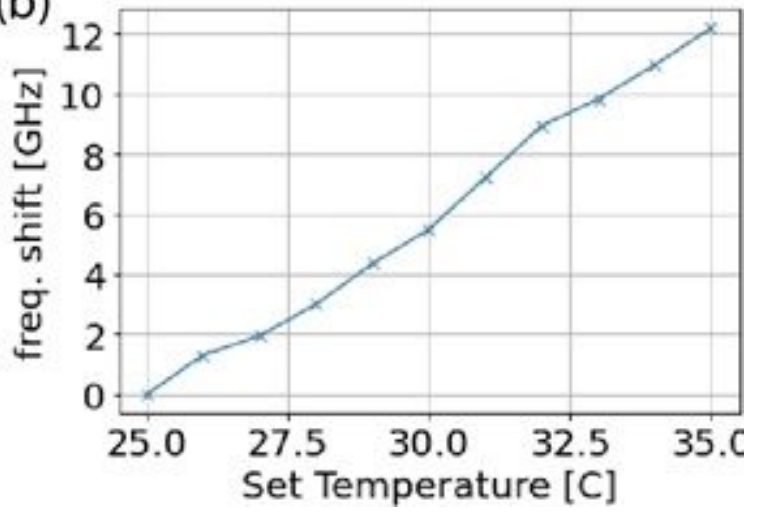

Figure 7

(a) Distributed profile of the temperature along the sensing fiber obtained by a continuous update of the reference trace. (b) scatter plot of the frequency shift vs. recorded temperatures with a thermometer. We observe a linear response with a sensitivity $1.2 \mathrm{GHz} / \mathrm{K}$. 